\title{
TREATMENT OF “HOFFA” FRACTURES OF THE DISTAL FEMORAL CONDYLE
}

\author{
Prakash C. Wali ${ }^{1}$, Satish S. Nesari ${ }^{2}$
}

${ }^{1}$ Associate Professor, Department of Orthopaedics, Belagavi Institute of Medical Sciences, Belagavi, Karnataka. ${ }^{2}$ Assistant Professor, Department of Orthopaedics, Belagavi Institute of Medical Sciences, Belagavi, Karnataka.

\section{ABSTRACT}

\section{BACKGROUND}

Coronal plane "Hoffa" fractures of the distal femoral condyles are a rare entity. Lateral fractures are three times more common than medial fracture. They are commonly associated with supracondylar fractures of the distal femur; however, as an isolated injury they are rare.

\section{MATERIALS AND METHODS}

At our Institute during a three-year period, 05 cases of Hoffa fracture were treated. All the fractures were involving lateral condyle. The fracture were approached using a parapatellar incision. The parapatellar arthrotomy was carried out accordingly on the lateral side.

\section{RESULTS}

All fractures united within 4 months. There were no instances of infection or non-union. The mean range of motion was - Extension to 130 degrees of flexion. The average Tegner Lysholm knee score was 88. All patients achieved satisfactory joint function and regained their walking ability with good clinical results.

\section{CONCLUSION}

Direct reduction and stable fixation of Hoffa fractures leads to good clinical and radiological outcomes of these rare injuries. The parapatellar allows excellent visualisation and enough space for fixation of these fractures.

\section{KEYWORDS}

Hoffa's Fracture, Screw Fixation.

HOW TO CITE THIS ARTICLE: Wali PC, Nesari SS. Treatment of "Hoffa" fractures of the distal femoral condyle. J. Evolution Med. Dent. Sci. 2017;6(17):1383-1385, DOI: 10.14260/Jemds/2017/301

\begin{abstract}
BACKGROUND
Coronal plane Hoffa fractures of the femoral condyle are rare injuries first reported by Hoffa in 1904.[1] By definition, Hoffa fractures are intraarticular and the principles of their treatment parallel those of other intraarticular fractures anatomic reduction with fracture fixation secure enough to allow functional after treatment. Non-operative treatment of unicondylar femur fractures including Hoffa fractures yields poor results.[2,3] Generally, according to the location of the injury the incisions have been described. For lateral injuries a direct lateral approach and for medial injuries a medial approach. Starr et al described an extensive swashbuckler approach for addressing these fractures. ${ }^{[4]}$ There is paucity of data recommending which surgical approach and what implants and in which configuration are the most appropriate. We have utilised a parapatellar incision for all Hoffa fractures with parallel screw fixation perpendicular to the fracture. The purpose of this study was to evaluate the clinical outcome of this rare injury.
\end{abstract}

Financial or Other, Competing Interest: None.

Submission 20-01-2017, Peer Review 13-02-2017,

Acceptance 19-02-2017, Published 27-02-2017.

Corresponding Author:

Dr. Prakash C. Wali,

House No. 326,

CTS No. 5090/12

$2^{\text {nd }}$ Stage Hanumannagar,

Belgavi-19,

Karnataka.

E-mail: pakwali@yahoo.com

DOI: $10.14260 /$ jemds $/ 2017 / 301$

\section{MATERIALS AND METHODS}

Five adult patients with Hoffa fractures were treated using a parapatellar approach during a three-year period from 2013 to 2016. There were 4 males and 1 female patient. All five patients had lateral condyle fractures. All patients were evaluated with standard radiographic AP and lateral views for the knee.

\section{Surgical Technique}

The patient is positioned in supine position. Tourniquet is used during the surgery. Appropriate broad-spectrum antibiotics are administered before the surgery and continued for a period of $48 \mathrm{hrs}$. postoperatively. A parapatellar incision is taken just lateral to patellar border, and lateral parapatellar arthrotomy exposing the lateral condyle and the fracture. The fracture surfaces are cleaned and the displaced fragments anatomically reduced and fixed with bone clamps. We have found that the reduction is easier with the knee in 90-degree flexion and once the reduction is done we completely flex the knee which helps to maintain the reduction. Fixation is carried out first with cannulated 6.5 $\mathrm{mm}$ or $4 \mathrm{~mm}$ screws placed perpendicular to the fracture surfaces. Screws are inserted from the non-articular area of the femoral condyle antero-posteriorly. If screws are inserted from the articular surface, then countersinking of the screw head is carried out. Additional screws are placed in different directions to augment the fixation once compression is achieved, if required and primary fixation found not stable intraoperatively.

Postoperatively, an above knee slab was applied. Patients were followed every month till radiological union was seen and final x-ray at six months. Knee mobilisation was started 
at four weeks, initially passive and gradually active. Partial weight bearing was started at eight weeks and full weight bearing after radiological union. Return to pre-injury activities were advised at six months.

\section{RESULTS}

At 12 weeks, all fractures were healed clinically and radiographically. There were no superficial or deep infections. All patients had a documented range of motion of $0^{\circ}$ to at least $100^{\circ}$ at three months followup. At six months, all patients had 0 to 130 degrees flexion. Their average Knee Society score was 173 (average 160 - 180).

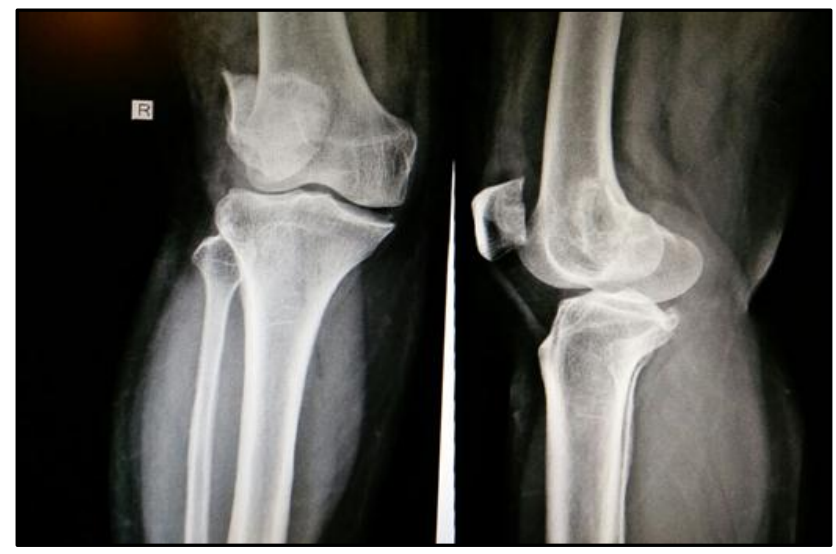

Figure 1. Preoperative X-Ray of Lateral Condyle Hoffa's Fracture

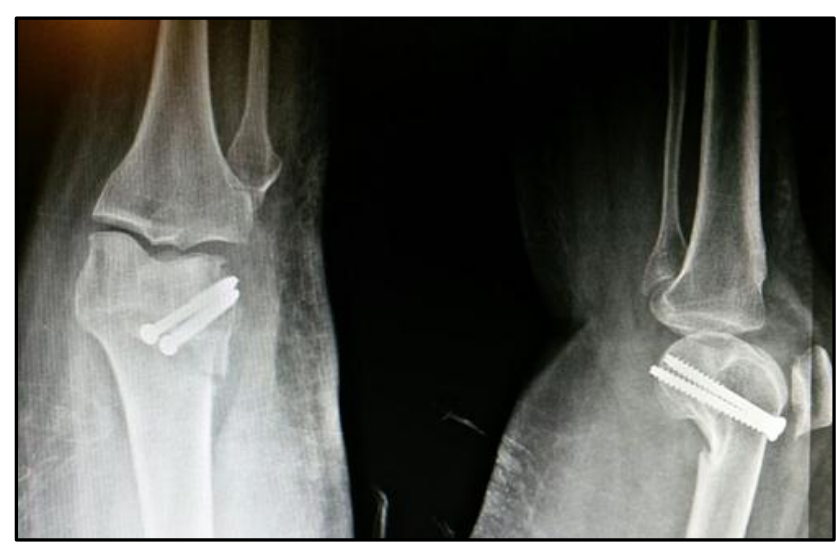

Figure 2. Post-Operative X-Ray Fixed with Interfragmentary Screws

\section{DISCUSSION}

Hoffa fractures are rare injuries and lateral fractures are more common than medial fractures. They usually occur as an isolated injury to the involved femur, but bilateral Hoffa fractures and unilateral bicondylar Hoffa fractures have been reported. In addition, Hoffa fractures associated with supracondylar intercondylar (AO/OTA 33C) femur fractures have been observed and the reporting authors highlight the need for preoperative detection of the Hoffa fracture to select the appropriate fixation method for the supracondylar fracture.

Examination invariably identifies effusion, but varus and valgus instability may be subtle. The neurovascular status should be assessed as in all high-energy knee injuries. Initial antero-posterior and lateral radiographs may be unimpressive, because Hoffa fractures especially when non- displaced are sometimes difficult to detect.(5) On the anteroposterior view, the foreshortened fractured condyle may lead to the appearance of varus or valgus malalignment.

Intra-articular coronal plane "Hoffa" fractures of the distal femur are rare injuries and difficult to treat. Conservative management often leads to unsatisfactory results and nonunion.(6) The long-term consequences of malunion, non-union and degenerative changes of a major joint require serious consideration. Open reduction and internal fixation is mandatory for good outcomes. Ostermann et al emphasised fracture compression and rigid internal fixation to allow immediate postoperative knee motion in their report on the long-term (average 68 months) results of 29 unicondylar femur fractures, of which there were six Hoffa fractures. They did not describe their surgical approach or the outcomes of the patients with Hoffa fractures, but they noted that 23 of 27 patients available for followup had an excellent result. In published literature, there has been no standardised surgical approach in treating these fractures except that by Holmes et al,(7) where they described an anterior midline approach with parapatellar arthrotomies according to the fracture location.

The Hoffa fractures are frequently associated with comminution at the articular surface. With the direct lateral or medial approaches, it is very difficult to address the comminution which is located in the central part of the distal femoral condyle with proximal migration of the distal fragment. There is an element of rotation of the femoral condyle, which is difficult to correct using the direct approaches. Liebergall et al(8) described a lateral approach to the knee using an osteotomy of Gerdy's tubercle in the treatment of a lateral coronal condylar fracture, but did not offer any outcome data on this single case. The authors argued that the osteotomy permits improved visualisation for open reduction and internal fixation. The direct parapatellar provides excellent visualisation of the fractures condyle, which makes it easier to address the problems of articular comminution and rotation. The standard method of fixing these fractures is using cannulated screws in compression mode. $(9,10)$

However, as these are shear fractures the parallel screw configuration sometimes leads to fixation failure. The parallel screw configuration perpendicular to the fracture line provides compression; however, in any case when the fixation is found to be not rigid and is not able to resist shear in comminuted fractures we had augmented our fixation using extra screws in multiple directions along with the compression screws to prevent displacement. This allows us to mobilise the knee in the early postoperative period without fear of loss of reduction. Two reports of arthroscopically assisted reduction and internal fixation of femoral condyle fractures have appeared.(11,12) Wallenbock and Ledinski(11) reported a series of 24 intraarticular knee fractures that they treated with arthroscopically assisted reduction and internal fixation, which included two Hoffa fractures.

Although noting that the technique of arthroscopically assisted reduction is demanding, they reported good early results. They did not describe specifically the technique or outcome of the patients with coronal fractures. McCarthy and Parker(12) described a patient with a sagittal plane lateral femoral condyle fracture. Although their fixation did not appear to achieve rigid compression, they cited reduced soft 
tissue dissection, blood loss and operative time and a faster recovery time after arthroscopic management as advantages over formal open reduction.

The drawbacks of our study were the limited number of patients. Also, there was no direct comparison with other modalities of treatment. As these fractures are a rarity, a multicentre trial comparing the outcomes using different approaches and different fixation techniques can ease the knowledge regarding this fracture.

\section{REFERENCES}

[1] Hoffa A. A Textbook of Fractures and DislocationsLehrbuch der Frakturen und Luxatnen. $4^{\text {th }}$ edn. Stuttgart: Ferdinand Enke-Verlag; 1904:p 453.

[2] Lewis SL, Pozo JL, Mairhead-Allwood WF. Coronal fractures of the lateral femoral condyle. J Bone Joint Surg Br 1989;71(1):118-20.

[3] Manfredini M, Gildone A, Ferrante R, et al. Unicondylar femoral fractures: therapuetic strategy and long term results. A review of 23 patients. Acta Orthop Belg 2001;67(2):132-8.

[4] Starr AJ, Jones AL, Reinert CM. The 'Swashbuckler': a modified anterior approach for fractures of the distal femur. J Orthop Trauma 1999;13(2):138-40.

[5] Allmann $\mathrm{KH}$, Altehoefer C, Wildanger G, et al. Hoffa fracture-a radiologic diagnostic approach. J Belge Radiol 1996;79(5):201-2.
[6] McDonough PW, Bernstein RM. Nonunion of a Hoffa fracture in a child. J Orthop Trauma 2000;14(7):51921.

[7] Holmes SM, Bomback D, Baumgaertner MR. Coronal fractures of the femoral condyle: a brief report of five cases. J Orthop Trauma 2004;18(5):316-9.

[8] Liebergall M, Wilber JH, Mosheiff R, et al. Gerdy's tubercule osteotomy for the treatment of coronal fractures of the lateral femoral condyle. J Orthop Trauma 2000;14(3):214-5.

[9] Hak DJ, Nguyen J, Curtiss S, et al. Coronal fractures of the distal femoral condyle: a biomechanical evaluation of four internal fixation constructs. Injury 2005;36(9):1103-6.

[10] Jarit GJ, Kummer FJ, Gibber MJ, et al. A mechanical evaluation of two fixation methods using cancellous screws for coronal fractures of the lateral condyle of the distal femur (OTA type 33B). J Orthop Trauma 2006;20(4):273-6.

[11] Wallenbock F, Ledinski C. Indications and limits of arthroscopic management of intra-articular fractures of the knee joint. Aktuelle Traumatol 1993;23(2):97101.

[12] McCarthy JJ, Parker RD. Arthroscopic reduction and internal fixation of a displaced intraarticular lateral femoral condyle fracture of the Knee. Arthroscopy 1996;12(2):224-7. 